Ann. Biol. anim. Bioch. Biophys., 1978, 18 (5), 1181-1195.

\title{
Etude quantitative de l'absorption et de la sécrétion du phosphore total et du phosphore phospholipidique dans le tube digestif du Mouton
}

\author{
par A. THEWIS, E. FRANÇOIS *, Marie-France THIELEMANS * \\ avec la collaboration technique de F. BURNY, F. DUPONT * et L. LALLEMAND * \\ Chaire de Physiologie animale ef de Zoolechnie, \\ Faculté des Sciences Agronomiques de l'Etat, \\ * Ministère de l'Agriculture, \\ Centre de Recherches agronomiques de l'Etat, \\ Station de Chimie ef de Physique agricoles, \\ 5800 Gembloux, Belgique.
}

Summary. Absorption and secretion of total and phospholipid phosphorus in the gastroinfestinal tract of the sheep : A quantitative study.

Quantitative aspects of the absorption and the secretion of total and lipid phosphorus were studied in adult wethers fed chopped meadow hay using radiocerium and chromium sesquioxide-paper as unabsorbable reference markers. Radiocerium was administered with the hay uniformly labelled in its totality, whereas identical doses of chromium sesquioxide-paper were distributed reliably and simply by incorporation into alfalfa pellets (mode of preparation to be described in another publication). The chopped labelled hay and chromium sesquioxide pellets were fed twice a day. High $P$ secretion was found in the reticulo-rumen, ranging from 2.5 to $10 \mathrm{~g}$ a day and consisting mainly $(90 \mathrm{p} .100)$ of mineral $P$. The organic $P$, probably of microbial origin, represented a daily secretion of $15 \mathrm{~g}$ of phospholipids. Considerable total and phospholipid P secretion occurred in the first part of the small intestine (ranging from 6.2 to $48.6 \mathrm{~g}$ of total $\mathrm{P}$ and 1.8 to $3.4 \mathrm{~g}$ of phospholipid $\mathrm{P}$ a day). Biliary phospholipids amounted to 30 p. 100 of total $P$ secretion, and phosphates to the remainder. This secretion of total and lipid $P$ was followed in all subjects by absorption of comparable intensity in the small intestine. As far as the present experimental conditions are concerned, these conclusions are not compatible with the existence of a saturable process for $\mathbf{P}$ absorption.

\section{Introduction.}

Les transferts des substances minérales et organiques à travers la paroi digestive sont intenses ef très complexes. C'est pourquoi l'étude qualitative et quantitative de l'absorption et de la sécrétion des molécules tout au long du tractus digestif ainsi que les mécanismes qui régissent leur transfert au niveau de la muqueuse gastrointestinale restent souvent mal connus. Les méthodes d'investigation dans ce domaine peuvent 
être classées en 3 catégories : techniques in vitro (anses intestinales dévaginées), in situ (anses intestinales isolées) et enfin in vivo. Il s'agit dans ce dernier cas, soit d'animaux porteurs de fistules et recevant ou non un marqueur indigestible soit de sujets intacts abattus après marquage uniforme des digesta à l'aide d'un traceur non absorbé.

Si la première méthode est souvent critiquée, - la présence de canules étant susceptible de modifier le transit des digesta - la seconde par contre, ne rend compte que de la situation observée au moment de l'abattage. D'autre part, lorsque dans un même organe, absorption et sécrétion coexistent, cette dernière technique donne simplement la résultante des échanges. Une étude critique des différentes méthodes a été publiée par Miller (1972).

Dans le présent travail, les transferts de phosphore total $\left(P_{T}\right)$ et de phosphore phospholipidique $\left(P_{P L}\right)$ dans le tube digestif du Mouton sont étudiés sur des animaux abattus à l'équilibre de marquage réalisé au moyen d'un marqueur non absorbé. Si au cours des expériences décrites plus loin, deux traceurs différents sont utilisés, ce fait doit être attribué uniquement aux objectifs multiples de l'expérimenta tion.

Le peu de données disponibles tant sur la localisation que sur la quantification des transferts de ces substances chez les ruminants est à la base de nos travaux. En effet, les diverses recherches actuellement publiées portent plutôt sur la détermination de la digestibilité réelle du phosphore en estimant les pertes endogènes fécales par la méthode de la dilution isotopique du radiophosphore (Compère, 1967 ; Guéguen, 1962 ; Kleiber ef al., 1951). Seuls Ben-Ghedalia ef al. (1975), Bruce et al., (1966), François (1974), Smith ef al. (1955) chez le Mouton, Chandler ef Cragle (1962), Yang et Thomas (1965) chez le Veau et enfin Rogers et van't Klooster (1969) et Smith et al. (1956) chez la Vache ont tenté de localiser les sites d'absorption et de sécrétion du phosphore, voire même d'estimer l'importance des transferts. A la suite de ces travaux, il apparaît que le rumen est le siège d'une importante sécrétion de phosphore d'origine salivaire tandis que l'absorption de cet élément a lieu essentiellement dans l'intestin grêle.

Enfin, en ce qui concerne la dynamique digestive des phospholipides chez le Ruminant, seuls Kurilov et Firsov (1974) signalent une synthèse de ces composés dans le rumen tandis que Lennox, Lough et Garton (1968) ont montré une absorption progressive des phospholipides essentiellement d'origine biliaire au niveau du jéjunum et de l'iléon du Mouton.

\section{Matériel expérimental et techniques utilisées.}

\section{1. - Déroulement des expériences.}

Expérience 1 . - En vue de suivre l'évolution des quantités de $\mathrm{P}$ total transférées tout au long du tube digestif du Mouton, sept béliers adultes placés en cage à métabolisme reçoivent du foin de prairie haché uniformément marqué au radiocérium. La ration est distribuée à 9 h 00 et 16 h 00 . Les animaux disposent d'eau de boisson à volonté. Les consommations de matière sèche, les poids des animaux ef les indices d'ingestion sont donnés au tableau 1 . Etant donné que la vitesse de transit influence les phénomènes 
étudiés, on synchronise tous les animaux à ce point de vue en fixant un indice d'ingestion unique $\left.{ }^{(}\right)$(François et Compère, 1972).

TABLEAU 1

Poids, consommation ef indice d'ingestion (Exp. I)

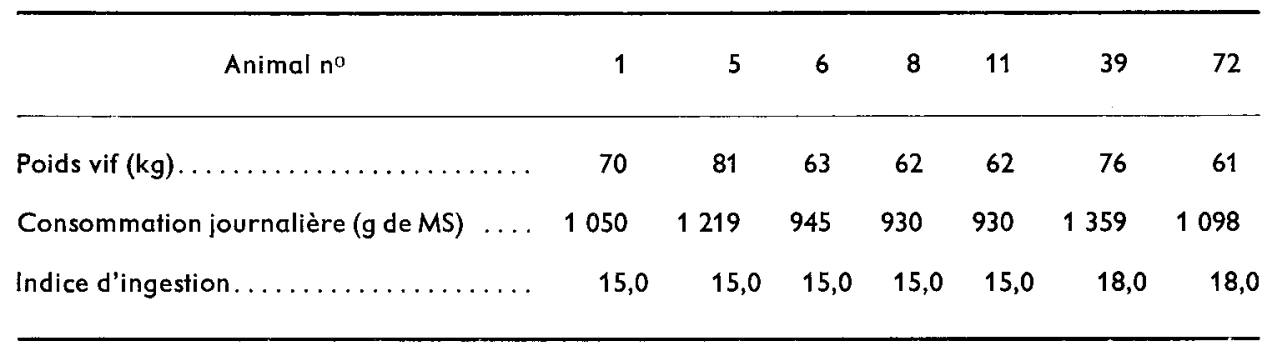

Après 6 ou 7 jours de ce régime, les matières fécales sont récoltées régulièrement afin de s'assurer de la qualité du marquage. Les animaux sont abattus entre 11 et 15 jours après la distribution du premier repas marqué. Les abattages ont lieu environ 6 h 30 après la présentation du dernier repas. Le tube digestif est immédiatement prélevé et vidé de son contenu.

Expérience 11 . - Trois béliers adultes placés en cage à métabolisme reçoivent pendant une quinzaine de jours du foin de prairie haché en vue d'étudier simultanément les transferts de $P_{\mathrm{T}}$ et de $\mathrm{P}_{\mathrm{LP}}$. L'aliment est distribué en deux fois à $9 \mathrm{~h} 00$ et $16 \mathrm{~h} 00$. Les animaux disposent d'eau à volonté. Avant chaque repas, les animaux reçoivent une dose de pellets de luzerne contenant du papier imprégné d'oxyde de chrome (Corbett, Greenhalgh et MacDonald, 1958). La validité de l'oxyde de chrome-papier comme marqueur a été démontrée par ailleurs par Thill et al. (1978). Les doses de $25 \mathrm{~g}$ de pellets contiennent une quantité identique de $\mathrm{Cr}_{2} \mathrm{O}_{3}$ soit environ $2 \mathrm{~g}$. L'identité des doses a été vérifiée. Les poids des animaux, les quantités de foin consommées et les indices d'ingestion sont donnés au tableau 2.

TABLEAU 2

Poids, consommation ef indice d'ingestion (Exp. II)

\begin{tabular}{|c|c|c|c|}
\hline Animal no & 62 & 57 & 55 \\
\hline Poids vif $(\mathrm{kg})$. & 44,1 & 35,9 & 37,7 \\
\hline Consommation journalière ( $g$ de $M S$ )..... & 1235 & 1005 & 1056 \\
\hline Indice d'ingestion & 28 & 28 & 28 \\
\hline
\end{tabular}

(1) Indice d'ingestion $\frac{\text { MS consommée }(g)}{\text { Poids de l'animal }(g)} \times 1000$. 
Après 7 jours de distribution du marqueur, les fèces sont récoltées toutes les $2 \mathrm{~h}$ pendant $48 \mathrm{~h}$ et une fois par jour pendant 4 jours. Les animaux sont ensuite abaftus à jeun; le tube digestif est prélevé, les contenus sont recueillis, traités et analysés comme décrit plus loin.

\section{2. - Marquage uniforme des contenus gastrointestinaux.}

Le radiocérium est donc administré aux animaux sous forme de foin uniformément marqué. Le marquage uniforme de la masse de foin nécessaire pour nourrir un animal pendant la durée de l'expérience est réalisé suivant la technique décrite par François et Thewis (1976).

Pour l'oxyde de chrome-papier, une forme d'administration sûre et commode a été mise au point (Thill et al., 1978). Découpé en petits morceaux, le papier est incorporé à du foin de luzerne haché ef l'ensemble est condensé au moyen d'une presse à pellets de laboratoire. Les pellets obtenus sont facilement acceptés par les animaux.

L'aliment uniformément marqué au ${ }^{144} \mathrm{Ce}$ et les pellets à l'oxyde de chromepapier sont distribués deux fois par jour. Après 6 à 7 jours de ce régime, on récolte les matières fécales pendant une dizaine de jours afin de vérifier la qualité du marquage. Les animaux sont ensuife abattus. Les échantillons de matières fécales sont séchés à $100^{\circ} \mathrm{C}$ jusqu'à poids constant. Ils sont ensuite finement moulus (tamis de $0,25 \mathrm{~mm}$ ) en vue du dosage du ${ }^{144} \mathrm{Ce}$ et $\mathrm{du} \mathrm{Cr}_{2} \mathrm{O}_{3}$.

C'est donc l'évolution de la concentration fécale en radio-traceur qui nous sert à tester l'uniformité du marquage. En effet, il semble raisonnable d'admettre qu'une faible variabilité de ce paramètre dans le temps est la traduction d'une régularité équivalente aux différents niveaux du tube digestif.

\section{3. - Abattage des animaux ef traitement des contenus gastrointestinaux.}

Les animaux sont abattus à des temps variables après la présentation du dernier repas, l'abattage se fait au revolver; une saignée complète de l'animal est ensuite pratiquée. La paroi abdominale est incisée. Des ligatures sont pratiquées à l'entrée et à la sortie des estomacs et du cæcum ef tous les $50 \mathrm{~cm}$ environ le long des intestins, afin d'éviter les déplacements du contenu. Les 4 estomacs sont dissociés. L'intestin grêle est divisé en 4 ou 5 parties d'égale longueur. Le cæcum est isolé du reste du gros intestin qui est également divisé en 4 segments. Le vidage des intestins se fait le plus tôt possible après l'abattage : l'ensemble des opérations dure une heure environ. La matière est recueillie dans des récipients recouverts de Téflon et préalablement tarés. Elle fait l'objet d'une détermination de la matière sèche ef subit ensuite une mouture fine (tamis de $0,25 \mathrm{~mm}$ ). Cependant les échantillons destinés au dosage du $P_{P L}$ sont stockés, après traitement dans un bain-marie à $100^{\circ} \mathrm{C}$ pendant une demiheure, à $-20^{\circ} \mathrm{C}$ et sous azote dans des récipients hermétiquement fermés.

\section{4. - Déterminations analytiques.}

Dosages radiochimiques. - Le radiocérium est dosé dans les matières fécales, agglomérées en disques à la presse, par son émission bêta comme décrit par Thewis, François et Thill (1975). Toutefois, en vue d'obtenir des disques plus résistants, on ajoute de la caséine à raison de $0,5 \mathrm{~g}$ pour $4,5 \mathrm{~g}$ de matière fécale radioactive pulvé- 
rulente ; après addition de quelques gouttes d'eau, on homogénéise parfaitement et on soumet au pressage. Les échantillons d'aliment, des divers contenus digestifs et de fèces prélevées juste avant l'abattage - tous de composition fort différente doivent être dilués préalablement avec une matière pulvérulente non radioactive, des fèces par exemple. La mesure correcte de l'émission bêta ne peut en effet se faire qu'à partir d'échantillons de structure physique pratiquement identique. On mélange donc intimement après addition de quelques gouttes d'eau $0,5 \mathrm{~g}$ de l'échantillon à analyser, $4 \mathrm{~g}$ de fèces non radioactives finement moulues ef $0,5 \mathrm{~g}$ de caséine. On soumet ensuite au pressage et on compte. Le détecteur utilisé (1) à fenêtre mince, de $5 \mathrm{~cm}$ de diamètre, est du type à courant gazeux; le mouvement propre de l'instrument est de l'ordre d'un choc par minute.

Dosages chimiques.

- Oxyde de chrome : Il est dosé dans l'aliment, les contenus gastrointestinaux et les fèces par titrage après oxydation nitro-perchlorique (François, Thill et Thewis, 1978).

- Extraction des phospholipides : Ils sont extraits des aliments ef des contenus digestifs par épuisement à l'aide des solvants suivants : éthanol, chloroforme, chloroformeméthanol $(2: 1 \mathrm{v} / \mathrm{v})$, chloroforme-méthanol-acide nitrique $(645: 322: 33 \mathrm{v} / \mathrm{v} / \mathrm{v})$.

Les différents extraits sont combinés et lavés à l'aide d'une solution de $\mathrm{NaCl}$ selon Folch, Lees et Stanley (1957). Les phospholipides se retrouvent alors dans la phase chloroformique qui est recueillie et évaporée à l'aide d'un évaporateur rotatif à $45^{\circ} \mathrm{C}$ et sous vide. Le résidu subit ensuite une oxydation nitro-perchlorique (Thewis, François et Thielemans, 1978).

- Dosage du phosphore : $\mathbf{P}_{\mathrm{T}}$ est dosé dans la solution issue de la minéralisation nitroperchlorique de 1 ou $2 \mathrm{~g}$ de matière finement moulue. $P_{P L}$, quant à lui, est dosé dans la solution issue de la minéralisation de l'extrait chloroformique obtenu ci-dessus. Suivant la concentration en phosphore dans la solution, on utilise la méthode au bleu de molybdène préconisée par Fogg ef Wilkinson (1958) - échantillons contenant de 1 à 20 ppm dans la solution - ou celle au jaune de vanadomolybdate (Misson, 1908) - échantillons de concentration supérieure à 20 ppm dans la solution -. Dans les deux cas, les dosages sont effectués à l'aide d'un analyseur automatique ${ }^{2}$ ). Nous avons vérifié la bonne concordance entre les deux méthodes sur plusieurs échantillons.

5. - Traitement des données.

Le poids de $P$ sécrété ou absorbé dans chaque organe ou section du tube digestif peut être calculé au moyen de la formule suivante :

$$
P=M_{24}\left(\frac{e_{c}}{m_{c}}-\frac{e_{c+1}}{m_{c+1}}\right)
$$

où $M_{24}$ représente la dose de marqueur ingérée journellement; $e_{c}$ et $e_{c+1}$ représentent la concentration en $P$ en $\mu \mathrm{g} / \mathrm{g}$ de matière sèche du contenu de deux segments succes-

(1) Nuclear Chicago-Searle.

(2) Technicon, Autoanalyzer II, Tarrytown, New York.

Annales de Biologie animale. - 1978 
sifs (sens oral-aboral); $\mathrm{m}_{\mathrm{c}}$ et $\mathrm{m}_{\mathrm{c}+1}$ représentent la concentration en marqueur exprimée en $\mathrm{CPM} / \mathrm{g}$ de matière sèche de ces mêmes contenus. Cette expression exploite simplement le caractère non résorbable du marqueur et le fait que la dose journalière doit passer dans son intégralité en chaque point du tractus digestif.

Il est clair qu'elle étend à toute la journée la situation observée au moment de l'abattage, Dès lors, l'exactitude des résultats est étroitement liée à la variation du rapport entre $P$ et le marqueur aux différents niveaux du tube digestif et au cours de la journée. De plus, elle ne permet pas de distinguer absorption et sécrétion lorsque les deux courants coexistent. Par contre, contrairement à la technique des fistules, celle des abattages permet de bien tenir compte de la vitesse de transit des digesta dans les différents segments ; cet apport de la technique est évidemment appréciable car comme nous l'avons rappelé plus haut le transit constitue l'un des principaux facteurs qui règlent l'absorption des molécules.

En conséquence, la technique des abattages appliquée à un nombre imporlant d'animaux abattus à divers moments par rapport à l'heure de distribution du repas nous paraît être la méthode de choix pour étudier ces problèmes d'échanges à travers la paroi intestinale. Elle a d'ailleurs déjà été largement utilisée par Hurwitz et Bar $(1965,1970)$ chez la Poule pondeuse et le Poulet pour étudier l'absorption et la sécrétion du P. Cette méthode a également été employée dans diverses espèces animales pour déterminer les sites d'absorption et de sécrétion de différents éléments minéraux à savoir le calcium, le phosphore (Chandler et Cragle, 1962 ; Schryver et al., 1970) et le magnésium (Guenter et Sell, 1973).

\section{Résultats.}

Les quantités journalières de $P_{\mathbf{T}}$ apparemment sécrétées ou absorbées dans le tube digestif sont données au tableau 3 pour les 7 béliers de la première expérience et au tableau 5 pour les 3 animaux de la seconde.

Au niveau du réticulo-rumen, dans tous les cas on observe un apport de $P_{T}$ très important, essentiellement d'origine salivaire. Il varie de $2,8 \mathrm{à} 9,6 \mathrm{~g}$ de $\mathrm{P}$ par animal et par jour. Le feuillet est le siège d'une absorption nettement marquée dans tous les cas. En ce qui concerne la caillette, on note tantôt une absorption tantôt une sécrétion. L'intestin grêle est le siège d'une sécrétion massive de $\mathrm{P}$ atteignant dans certains cas $48 \mathrm{~g}$ par jour et par animal. On constate que, dans tous les cas, cette sécrétion massive de $\mathrm{P}_{\mathrm{T}}$ dans le premier quart de l'intestin grêle est suivie d'une absorption quasi équivalente dans le reste de cet organe. Elle se poursuit jusque dans le cæcum. Les échanges de $P_{T}$, tout en restant notables, ont une intensité relativement moindre dans le reste du gros intestin ; un tronçon donné peut être le siège d'une absorption prédominante chez certains sujets et d'une sécrétion chez d'autres.

Nous donnons au tableau 4 la répartition moyenne du $P_{T}$ ef du $P_{P L}$ dans le tractus digestif des moutons de la seconde expérience. Comme on peut le constater, la fraction de $P_{P L}$ est assez peu importante ; elle atteint son maximum au niveau du premier tronçon de l'intestin grêle.

Les quantités journalières de $P_{P L}$ absorbées ou sécrétées tout au long du tube digestif sont données au tableau 5 pour les 3 moutons. On remarque que le rumen, la caillette et le premier quart de l'intestin grêle sont les zones de sécrétion nette des phos- 
1187

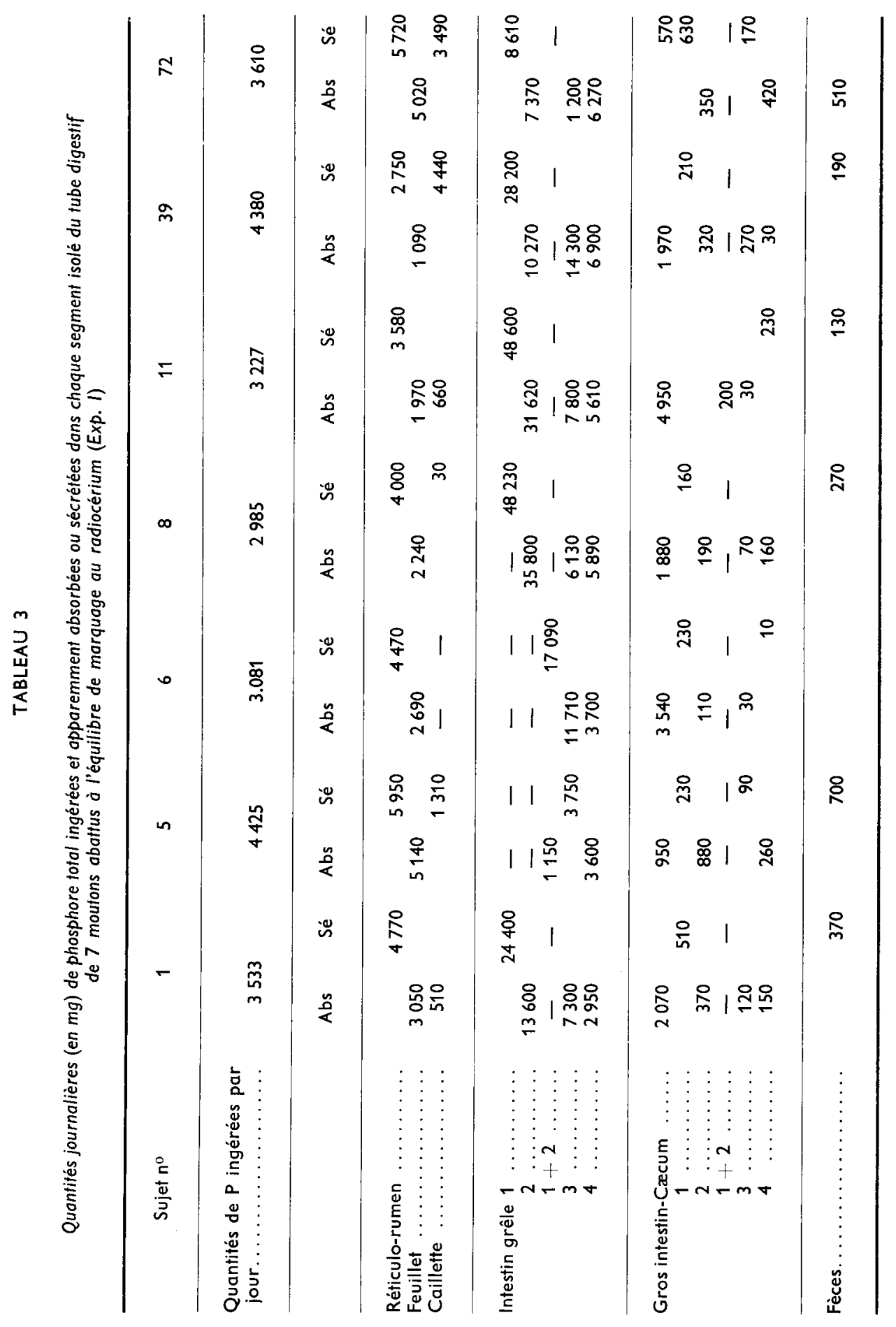


pholipides. La sécrétion de $P_{P L}$ représente environ 7 p. 100 de la sécrétion de $P_{T}$ dans le réticulo-rumen et 30 p. 100 de l'apport endogène de $P_{T}$ dans le premier tronçon de l'intestin grêle. Quant à l'absorption, elle se situe au niveau du feuillet, des 3 derniers quarts de l'intestin grêle et du cæcum. L'importante sécrétion de $P_{P L}$ dans le premier segment de l'intestin grêle est suivie d'une absorption aboutissant à un bilan quasi nul dans l'intestin grêle. Dans la partie tubulaire du gros intestin, les échanges de P PL sont peu importants.

\section{TABLEAU 4}

Concentration moyenne en phosphore fotal $\left(\mathrm{P}_{\mathrm{T}}\right)$ et en phosphore phospholipidique ( $\mathrm{P}_{\mathrm{PL}}$ ) dans les contenus des différents segments du tube digestif de 3 moutons recevant du foin de prairie haché (Exp. II)

\begin{tabular}{|c|c|c|c|}
\hline & $\mathrm{P}_{\mathrm{T}}\left(\mathbf{(}^{\mathbf{1}}\right)$ & & $P_{P L}\left({ }^{1}\right)$ \\
\hline & $x \pm \sigma$ & $x \pm \sigma$ & En p. 100 du $P$ total \\
\hline Aliment & $1767 \pm 119$ & $51 \pm 4$ & 2,89 \\
\hline $\begin{array}{l}\text { Réticulo rumen } \ldots \ldots \ldots \\
\text { Fevillet } \ldots \ldots \ldots \ldots \ldots \ldots \\
\text { Caillette............. }\end{array}$ & $\begin{array}{l}7273 \pm 1021 \\
7547 \pm 1033 \\
6798 \pm 659\end{array}$ & $\begin{array}{l}455 \pm 41 \\
346 \pm 81 \\
502 \pm 68\end{array}$ & $\begin{array}{l}6,26 \\
4,58 \\
7,38\end{array}$ \\
\hline $\begin{aligned} & \text { Intestin grêle } 1 \ldots \ldots \ldots \\
& \ldots \ldots \ldots \\
& 2 \ldots \ldots \ldots \\
& 4 \ldots \ldots \\
& 4 \ldots \ldots \\
&\end{aligned}$ & $\begin{array}{l}9184 \pm 775 \\
6465 \pm 904 \\
6089 \pm 1033 \\
3816 \pm 158 \\
\end{array}$ & $\begin{array}{c}1979 \pm 173 \\
987 \pm 279 \\
501 \pm 69 \\
374 \pm 47\end{array}$ & $\begin{array}{r}21,55 \\
15,27 \\
8,23 \\
9,80\end{array}$ \\
\hline 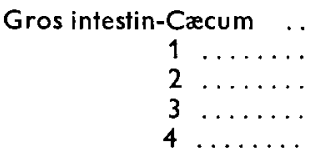 & $\begin{array}{l}3371 \pm 441 \\
2726 \pm 227 \\
2579 \pm 277 \\
2447 \pm 170 \\
2807 \pm 282\end{array}$ & $\begin{array}{l}233 \pm 21 \\
234 \pm 14 \\
237 \pm 54 \\
203 \pm 18 \\
188 \pm 20\end{array}$ & $\begin{array}{l}6,91 \\
8,58 \\
9,19 \\
8,30 \\
6,70\end{array}$ \\
\hline
\end{tabular}

(1) Les concentrations sont exprimées en ppm dans la matière sèche.

\section{Discussion et conclusions.}

1. - Réticulo-rumen. - La sécrétion importante de phosphore total observée dans le rumen est confirmée par les observations de Smith ef al. (1955), Bruce et al. (1966) chez le Mouton et de Chandler et Cragle (1962) chez le Veau.

Elle s'explique par l'apport salivaire de $P$ très important chez le ruminant. En effet, selon Kay (1966), le volume de salive sécrétée par un mouton consommant un régime de foin et de concentré serait compris entre 6 ef 16 litres par jour. Selon Wilson (1963), la production de salive parotidienne chez un mouton recevant du foin haché s'élèverait à 8-9 litres par jour. Si par ailleurs, on admet avec McDougall (1948), Dukes (1947), Emery et al. (1960) et Bailey ef Balch (1961) que la salive des ruminants contient de 0,3 à $1 \mathrm{~g}$ de $P$ par litre, on constate que la seule sécrétion salivaire rend aisément compte de l'apport endogène constaté au niveau du rumen.

Cependant, certains auteurs (Smith et al., 1955) postulent l'existence d'un transfert direct de $P$ du sang vers le contenu du rumen à travers la paroi, qui représente- 


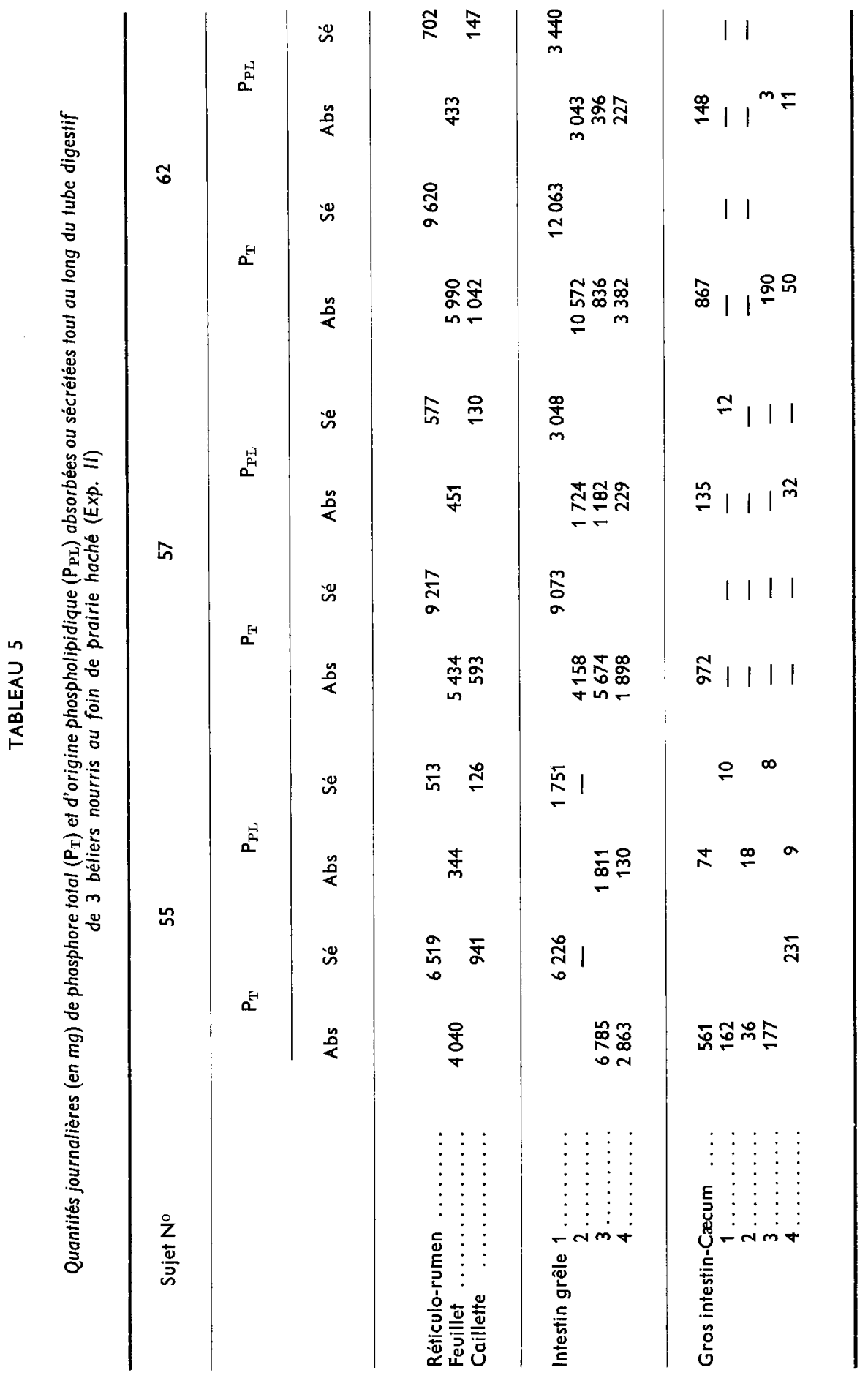


rait 50 p. 100 de la sécrétion endogène. Parthasarathy, Garton et Phillipson (1952) ont, par ailleurs, montré in vivo que la paroi du rumen est perméable au phosphore dans les deux directions.

Quoiqu'il en soit, la technique des marqueurs non résorbables ne nous donne, pour chaque organe ou segment, qu'une valeur résultante, somme algébrique des deux flux opposés : absorption et sécrétion. Elle ne nous permet pas de mettre en évidence au niveau du rumen une absorption masquée par une sécrétion plus importante.

La sécrétion de phospholipides observée dans le rumen de nos moutons s'élèverait à environ $15 \mathrm{~g}$ par animal et par jour si l'on tient compte d'un facteur de conversion de 25 préconisé par Adams ef Heath (1963). Cet apport phospholipidique confirme les observations de Kurilov et Firsov (1974). Ces auteurs pensent que ces phospholipides sont essentiellement d'origine microbienne. En effet, les phospholipides sont la classe lipidique la mieux représentée dans le rumen de moutons recevant un régime dépourvu de graisses. Ils représentent environ 50 à 70 p. 100 des lipides microbiens (Kurilov ef al., 1976 ; Keeney, Katz el Allison, 1962 ; Katz et Keeney, 1966). De plus, Patton, Mc Carthy et Griel (1970) ont mis en évidence in vitro une synthèse de lipides polaires par les protozoaires du rumen. Par ailleurs, la salive pourrait constituer une source de phospholipides. Leclercq et Di Costanzo (1976) ont, en effet, montré que la glande sous-maxillaire du rat contient 34 p. 100 de lipides dont 59 p. 100 seraient sous forme de phospholipides.

Selon les premières données dont nous disposons, l'apport de $P_{P L}$ dans le rumen ne représenterait que 7 p. 100 de la sécrétion de $P_{T}$. La sécrétion salivaire et peut-être le passage direct à travers la paroi du rumen constitueraient donc les sources essentielles de $P$ endogène au niveau de cet organe.

2. - Feuillet. - L'absorption de $P_{\mathrm{T}}$ observée au niveau du feuillet confirme les observations de Chandler ef Cragle (1962) chez les veaux de 20 semaines. Elle ne concorde cependant pas avec les résultats rapportés par Smith et al. (1955). Les phospholipides seraient également absorbés au niveau du feuillet.

II est à craindre toutefois que nos valeurs soient exagérées. II se pourrait en effet que le triage exercé par le feuillet altère quelque peu la répartition des marqueurs dans les digesta en provenance du réticulo-rumen. Il est à remarquer de toute manière que le contenu du feuillet ne concerne qu'une faible fraction des digesta.

3. - Caillette. - Les résultats obtenus pour la caillette sont fort variables en ce qui concerne le $\mathrm{P}_{\mathrm{T}}$ : l'absorption pouvant l'emporter sur la sécrétion et vice versa. Chandler et Cragle (1962) tout comme Yang et Thomas (1965), constatent que la caillette est un site d'absorption du phosphore total chez le Veau. Par ailleurs, en ce qui concerne le $P_{P L}$ nous constatons une sécrétion chez les 3 béliers de la seconde expérience.

4. - Intestin grêle. - Les données enregistrées au niveau de l'intestin grêle revêtent un grand intérêt, tant en ce qui concerne le $P_{T}$ que le $P_{P L}$.

La sécrétion phosphorée observée dans le début de l'intestin grêle (łabl. 3 et 5 ) suivie d'une absorption dans le reste de l'organe, correspond aux observations de Chandler et Cragle (1962), de Yang ef Thomas (1965) chez le Veau, et de Hurwitz et Bar (1965) chez la Poule pondeuse. Travaillant sur des ruminants porteurs de fistules 
permanentes au niveau de l'intestin grêle, Bruce ef al. (1966) et Rogers ef van't Klooster (1969) localisent l'absorption du P dans le jéjunum et l'iléon. Par ailleurs, selon Ben-Ghedalia et al. (1975), c'est au niveau des 15 premiers mètres de l'intestin grêle que s'effectue l'absorption du phosphore.

Pour expliquer la sécrétion massive, qui n'a jamais été évaluée à notre connaissance, dans le premier tronçon de l'intestin grêle, on peut invoquer les sécrétions digestives déversées à ce niveau. L'absence de renseignements bibliographiques en ce qui concerne la composition phosphorée des sécrétions des glandes de Brünner et du suc pancréatique ne nous permet toutefois pas d'apprécier leur part d'intervention. En ce qui concerne la bile, elle apporterait quelque $500 \mathrm{mg}$ de $P$ par jour (Adams et Heath, 1963). Selon Heath et Hill (1969), $6 \mathrm{~g}$ de phospholipides biliaires seraient sécrétés journellement dans le duodénum. Si l'on admet avec Zamytchkina et Grodsensky (1955), que le $P$ biliaire est à 99 p. 100 sous forme de $P_{P L}$, on constate que les valeurs que nous avançons en ce qui concerne la sécrétion de $P_{P L}$ dans le premier quart de l'intestin grêle, sont nettement supérieures à celles mentionnées ci-dessus (tabl. 5).

II se pourrait que les valeurs numériques présentées pour évaluer les transferts de $P$ dans l'intestin grêle soient excessives : la quantité de phosphore présente à cet endroit au moment de l'abattage pourrait être supérieure à la moyenne journalière. Le marquage uniforme des digesta d'un animal muni de fistules multiples pourrait nous éclairer à ce sujet, notamment en suivant l'évolution journalière du rapport entre le phosphore et le marqueur indigestible. II n'en reste pas moins vrai que la sécrétion de $P_{P L}$ dans le duodénum et une partie du jéjunum ne rend pas compte et de loin, de la quantité de $P_{T}$ sécrété à ce niveau. Nos chiffres montrent qu'il y a là une sécrétion massive de phosphates dont l'origine ef la raison d'être ne peuvent être expliquées sur la base des expériences réalisées à ce jour. Comme on l'a dit plus haut, on ne connaît pas bien la composition minérale des sécrétions des glandes de Brünner et du suc pancréatique. Young, Lofgreen et Luick (1966) ont étudié et estimé par des méthodes indirectes la sécrétion endogène de $\mathrm{P}$ et de $\mathrm{Ca}$ dans le tube digestif du Mouton. Ces auteurs concluent à l'existence d'importantes sécrétions de $P$ dans le tractus gastrointestinal et au recyclage d'une part importante de ce $P$ endogène. Ils pensent que la sécrétion de $P$ joue un rôle important dans la régulation de l'homéostasie phosphorée. Les travaux de Tomas et Somers (1974) et de Tomas (1974 a, b) ont récemment montré très clairement le rôle de la sécrétion phosphorée d'origine salivaire dans le maintien de l'homéostasie phosphorée chez le Mouton.

Quolqu'il en soit, il est très intéressant de constater qu'à cette importante sécrétion, fait suite une absorption qui équilibre presque quantitativement le bilan dans l'intestin grêle et qui se poursuit jusque dans le caecum. Si nous reprenons les données obtenues pour 7 animaux des expériences I et II, sujets pour lesquels nous possédons des données complètes, nous constatons que, dans les limites de nos conditions expérimentales, la quantité de $\mathrm{P}_{\mathrm{T}}$ absorbée dans les 3 derniers tronçons de l'intestin grêle est à première vue une fonction linéaire de la quantité sécrétée journellement dans le premier segment (fig. 1). Il semble donc dans notre cas ne pas y avoir de processus limitant le prélèvement du phosphore par les cellules épithéliales de l'intestin dans le chyme ni de phénomène de saturation. Cela confirme les observations de Hurwitz et Bar (1972) chez le Poulet et plaiderait plutôt en faveur d'un processus de simple diffusion pour expliquer le mécanisme de transfert de $P$ à travers la muqueuse intesti- 
nale. Cependant, nos travaux ne permettent pas de préjuger du passage de la muqueuse au plasma sanguin : cette étape constituerait l'élément limitant dans le transport de $\mathrm{P}$ selon Guéguen et Rérat (1967) et Wasserman et Taylor (1973).

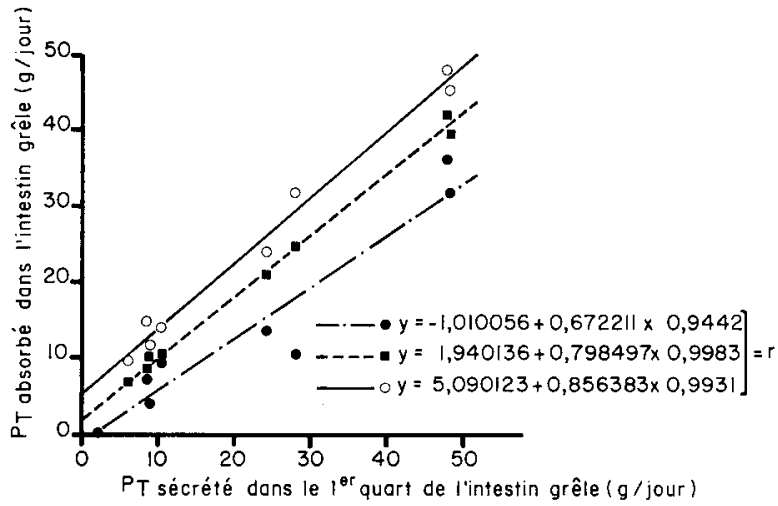

FIG. 1. - Evolution de l'absorption du $\mathrm{P}_{\mathrm{T}}$ dans les trois derniers quarts de l'intestin grêle du Mouton en fonction de la sécrétion observée dans le premier quart. $-\mathrm{P}_{\mathrm{T}}$ absorbé dans le deuxième quart de l'intestin grêle en fonction de la quantité sécrétée dans le premier quart ; - $\mathrm{P}_{\mathbf{T}}$ absorbé dans les deuxième et troisième quart de l'intestin grêle en fonction de la quantité sécrétée dans le premier quart ; $\circ \mathbf{P}_{\mathrm{T}}$ absorbé dans les trois derniers quarts de l'intestin grêle en fonction de la quantité sécrétée dans le premier quart.

On remarquera également que l'absorption progressive dans le jéjunum et l'iléon est tout aussi évidente pour le $P_{T}$ que $P_{P L}$ (tabl. 5). Le $P_{P L}$ est principalement absorbé dans les deuxième et troisième quarts de l'intestin grêle. Ulrich, Boucrot et Clément (1974) ont montré chez le Rat que les phospholipides étaient absorbés dans les 2 premiers tiers de l'intestin grêle. L'absorption progressive des phospholipides a également été observée par Lennox, Lough ef Garton (1968) chez le Mouton et par Sklan ef al. (1973) chez le Poulet. Ces auteurs constatent cependant une absorption plus rapide que dans notre cas. La reprise progressive des phospholipides pourrait très bien s'expliquer par l'existence d'un cycle entéro-hépatique des phospholipides analogue à celui suggéré par Boucrot (1972) chez le Rat. Toutefois, personne, à notre connaissance, n'a signalé l'existence d'une circulation entérohépatique des phospholipides chez les ruminants.

Par ailleurs, Leat et Harrison (1967), Lennox, Lough et Garton (1968) ef Arienti, Leat ef Harrison (1975) ont montré que dans l'intestin grêle du Mouton, la lécithine biliaire est progressivement hydrolysée en lysolécithine sous l'action des lipases pancréatiques activées par les sécrétions duodénales (glandes de Brünner). Cette lysolécithine favoriserait la solubilisation micellaire des acides gras mais également l'absorption des lipides par la muqueuse infestinale (Leat et Harrison, 1969 et 1974 ; Harrison et Leat, 1972 ; Lough et Smith, 1976). Cela expliquerait l'absorption des phospholipides essentiellement d'origine biliaire sécrétés dans le premier quart de l'intestin grêle.

5. - Gros intestin. - Dans le gros intestin, les transferts de $P_{T}$ sont encore importants. Hyden (1961) a également trouvé que des quantités considérables de phos- 
phates sont absorbées dans le cæcum. En ce qui concerne les phospholipides, on ne note plus guère d'échange à ce niveau.

En conclusion, nous avons pu confirmer et évaluer par la méthode des marqueurs de référence et des abattages la sécrétion de phosphore total dans le réticulo-rumen. Nous y avons observé un apport ou une synthèse non négligeable de phospholipides. Au niveau de l'intestin grêle, la technique a permis de mettre en évidence et d'évaluer la sécrétion massive de $P$ minéral et organique dans le premier quart, suivie d'une absorption quasi équivalente dans le reste de l'organe. L'examen de l'évolution des activités spécifiques des contenus et des sécrétions digestives par marquage des pools phosphorés de l'organisme au ${ }^{32} \mathrm{P}$, ainsi que l'emploi d'animaux fistulés, devraient nous permettre de préciser certains aspects de la sécrétion gastrointestinale du phosphore total et d'origine phospholipidique chez les petits ruminants.

Reçu en oout 1977.

Accepté en avril 1978.

Remerciements. - Ce travail a bénéficié de l'aide de l'Institut pour l'Encouragement de la Recherche Scientifique dans l'Industrie et l'Agriculture (IRSIA-CAMIRA)

\section{References}

ADAMS E. P., HEATH T. J., 1963. The phospholipids of ruminant bile. Biochim. biophys. Acta, 70, 688-690.

ARIENTI G., LEAT W. M. F., HARRISON F. A., 1975. The phospholipase activity of sheep pancreatic juice. Q. J. exp. Physiol., 60, 15-24.

BAILEY C. B., BALCH C. C., 1961. Saliva secretion and its relation to feeding in cattle. 2. The composition and rate of secretion of mixed saliva in the cow during rest. Br. J. Nutr., 15, 383-402.

BEN-GHEDALIA D., TAGARI H., ZAMWEL S., BONDI A., 1975. Solubility and net exchange of cal-. cium, magnesium and phosphorus in digesta flowing along the gut of the sheep. Br. J. Nutr., 33, 87-94.

BOUCROT P., 1972. Is there an entero-hepatic circulation of the bile phospholipids ? Lipids, 7, 282288.

BRUCE J., GOODALL E. D., KAY R. N. B., PHILLIPSON A. T., VOWLES L. E., 1966. The flow of organic and inorganic materials through the alimentary tract of the sheep. Proc. r. Soc. $B$, $166,46-62$.

CHANDLER P. T., CRAGLE R. G., 1962. Gastrointestinal sites of absorption and endogenous secretion of calcium and phosphorus in dairy calves. Proc. Soc. exp. Biol. Med., 111, 431-434.

COMPÈRE R., 1967. L'étude de l'équilibre phosphocalcique du régime basée sur les variations du métabolisme phosphoré. J. Duculot S. A., Gembloux, 132 pp.

CORBETT J. L., GREeNHALGH J. F. D., MacDONALD A. P., 1958. Paper as a carrier of chromium sesquioxide. Nature, 182, 1014-1016.

DUKES H. H., 1947. The physiology of domestic animals. Comstock Publ. Co, lthaca, New York, 6th ed., 817 Pp.

EMERY R. S., SMITH C. K., GRIMES R. M., HUFFMAN C. F., DUNCAN C. W., 1960. Physical and chemical changes in bovine saliva and rumen liquid with different hay-grain rations. J. Dairy Sci., 43, 76-80.

FOGG D. N., WILKINSON N. T., 1958. The colorimetric determination of phosphorus. Analyst, 83 406-414.

FOLCH J., LEES M., STANLEY G. H. S., 1957. A simple method for the isolation and purification of total lipids from animal tissues. J. biol. Chim., 226, 497-509.

FRANÇOIS E., 1974. La localisation de la résorption et de la sécrétion du phosphore dons le tractus digestif du mouton éfudiée au moyen des radiolanthanides. Thèse, Fac. Sci. Agron., Gembloux, Belgique. 
FRANÇOIS E., COMPÈRE R., 1972. Influence de la quantité ingérée sur le transit gastrointestinal et la digestibilité d'un foin de graminées long ou condensé chez le mouton, 301-319. In : Isotopes studies on the physiology of domestic animals. Proc. Symp. (Athens). Vienna IAEA.

FRANÇOIS E., THEWIS A., 1976. Le radiocérium utilisé comme élément de référence dans le tractus digestif du mouton. Réalisation d'un marquage régulier. Bull. Rech. agron. Gembloux, 11, 87100.

FRANÇOIS E., THILL N., THEWIS A., 1978. Une méthode rapide de dosage de l'oxyde de chrome dans les aliments, les fèces ef les contenus digestifs par titrage après oxydation nitroperchlorique. Ann. Zootech. (à paraître).

GUEGUEN L., 1962. L'utilisation réelle du phosphore du foin de luzerne par le Mouton, mesurée à l'aide de ${ }^{32} \mathrm{P}$. Ann. Biol. anim. Bioch. Biophys., 2, 143-149.

GUEGUEN L., RÉRAT A., 1967. Cinétique de l'absorption intestinale du phosphore chez le Porc. Ann. Biol, anim. Bioch. Biophys., 7, 39-46.

GUENTER W., SELL J. L., 1973. Magnesium absorption and secretion along the gastrointestinal tract of the chicken. J. Nutr., 103, 875-881.

HARRISON F. A., LEAT W. M. F., 1972. Absorption of palmitic, stearic and oleic acids in the sheep in the presence or absence of bile and/or pancreatic juice. J. Physiol., 225, 565-576.

HEATH T. J., HILL L. N., 1969. Dietary endogenous long-chain fatty acids in the intestine of sheep with an appendix on their estimation in feeds, bile and faeces. Austr. J. biol. Sci., 22, 1015-1029.

HURWITZ S., BAR A., 1965. Absorption of calcium and phosphorus along the gastrointestinal tract of the laying fowl as influenced by dietary calcium and egg shell formation. J. Nutr., 86, 433438.

HURWITZ S., BAR A., 1970. The sites of calcium and phosphate absorption in the chick. Poulf. Sci., 49, 324-325.

HURWITZ S., BAR A., 1972. Site of action of vitamin D. Am. J. Physiol., 222, 761-767.

HYDEN S., 1961. The use of reference substances and the measurement of flow in the alimentary tract, 35-45. In LEWIS D., Digestive physiology and nutrition of the ruminant. Butterworths, London.

KATZ I., KEENEY M., 1966. Characterisation of the octadecenoic acids in rumen digesta and rumen bacteria. J. Dairy Sci., 49, 962-966.

KAY R. N. B., 1966. The influence of saliva on digestion in ruminants. In : BOURNE G. H., World Rev. Nutr. Diet. 6, 292-325. S. Karger-Basel, Switzerland.

KEENEY M., KATZ I., ALLISON M. J., 1962. On the probable origin of some milk fat acids in rumen microbial lipids. J. am. Oil Chem. Soc., 39, 198-201.

KLEIBER M., SMITH A. M., RALSTON N. P., BLACK A. L., 1951. Radiophosphorus ( ${ }^{32} \mathrm{P}$ ) as tracer for measuring endogenous phosphorus in cow's faeces. J. Nutr., 45, 253-263.

KURILOV N. V., FIRSOV V. I., 1974. Effect of fats and synthetic fatty acids on lipid metabolism in the rumen. Vestn. S-Kh Nauk, Moscow, 2, $62-67$ (en russe) from Nutr. Abstr. Rev., 1975, 45, 4 (Abstr. $n^{\circ}$ 2051).

KURILOV N. V., SEVASTIANOVA N. A., KORSHUNOV V. N., MYSNIK N. D., PODSHIBYAKIN A. E., 1976. Amino acid and fatty acid composition of microorganisms passing into the abomasum of sheep. Livest. Prod. Sci., 3, 57-63.

LEAT W. M. F., HARRISON F. A., 1967. Effect of bile and pancreatic juice on the intestinal lipids of the sheep. Biochem. J., 105, 13 p.

LEAT W. M. F., HARRISON F. A., 1969. Lipid digestion in the sheep : effect of bile and pancreatic juice on the lipids of intestinal contents. Q. J. exp. Physiol., 54, 187-201.

LEAT W. M. F., HARRISON F. A., 1974. Origin and formation of lymph lipids in the sheep. Q. J. exp. Physiol., 59, 131-139.

LECLERCQ C., DI COSTANZO G., 1976. Métabolisme in vivo des phospholipides de la glande sousmaxillaire de rat. Biochimie, 58, 359-365.

LENNOX A. M., LOUGH A. K., GARTON G. A., 1968. Observations on the nature and origin of lipids in the small intestine of the sheep. Br. J. Nutr., 22, 237-246.

LOUGH A. K., SMITH A., 1976. Influence of the products of phospholipolysis of phosphatidylcholine on micellar solubilisation of fatty acids in the presence of bile salts. Br. J. Nutr., 35, 89-96.

MC DOUGALL E. I., 1948. Studies on ruminant saliva. I. The composition and output of sheep's saliva. Biochem. J., 43, 99-109. 
MILLER J. K., 1972. New techniques for intensive research with dairy cattle-mineral research. J. Dairy Sci., 55, 1211-1219.

MISSON G., 1908. Kolorimetrische Phosphorbestimmung im Stahl. Chemikerzeifung, $32,633$.

PARTHASARATHY D., GARTON G. A., PHILLIPSON A. T., 1952. The passage of phosphorus across the rumen epithelium of sheep. Biochem J., 52, XVI-XVII.

PATTON R. A., MC CARTHY R. D., GRIEL L. C. Jr., 1970. Lipid synthesis by rumen microorganisms. II. Further characterization of the effects of methionine. J. Dairy Sci., 53, 460-465.

ROGERS P. A. M., KLOOSTER van't A. Th., 1969. 3. The fate of Na, K, Ca, Mg and P in the digesta. Meded. LandbHogesch., Wageningen, 69, 26-38.

SCHRYVER H. F., CRAIG P. H., HINTZ H. F., HOGUE D. E., LOWE J. E., 1970 . The sife of calcium absorption in the horse. J. Nutr., 100, 1127-1132.

SKLAN D., BUDOWSKI P., ASCARELLI I., HURWITZ S., 1973. Lipid absorption and secretion in the chick : effect of raw soybean meal. J. Nutr., 103, 1299-1305.

SMITH A. H., KLEIBER M., BLACK A. L., BAXTER C. F., 1955. Transfer of phosphate in the digestive tract. II. Sheep. J. Nutr., 57, 507-527.

SMITH A. H., KLEIBER M., BLACK A. L., LOFGREEN G. P., 1956. Transfer of phosphate in the digestive tract. III. Dairy cattle. J. Nutr., 58, 95-112.

THEWIS A., FRANÇOIS E., THILL N., 1975. Le transit gastrointestinal chez le ruminant mesuré à l'aide des radiolanthanides. Signification des paramètres de la phase exponentielle des courbes de concentration fécale en traceur. Bull. Rech. agron. Gembloux, 10, 307-320.

THEWIS A., FRANÇOIS E., THIELEMANS M. F., 1978. Une méthode d'extraction quantitative des phospholipides totaux dans les aliments, les digesta ef les fèces. Ann. Biol. onim. Bioch. Biophys., 18, 645-650.

THILL N., FRANÇOIS E., THEWIS A., THIELEMANS M. F., 1978. Comparaison chez les petits ruminants de l'oxyde de chrome-papier et du radiocérium, marqueurs de la phase solide des digesta. Ann. Zootech. (à paraître).

TOMAS F. M., 1974a. Phosphorus homeostasis in sheep. Il. Influence of diet on the pathway of excretion of phosphorus. Aust. J. agric. Res., 25, 485-493.

TOMAS F. M., 1974b. Phosphorus homeostasis in sheep. III. Relationship between the amount of salivary phosphorus secreted and the quantities of phosphorus excreled via the urine and faeces. Aust. J. agric. Res., 25, 495-507.

TOMAS F. M., SOMERS M., 1974. Phosphorus homeostasis in sheep. I. Effect of ligation of parotid salivary ducts. Aust. J. agric. Res., 25, 475-483.

ULRICH CI., BOUCROT P., CLEMENT J., 1974. Site of biliary phospholipid absorption in the small intestine of the rat. Biochimie, 56, 429-434.

WASSERMAN R. H., TAYLOR A. N., 1973. Intestinal absorption of phosphate in the chick : effet of vitamin $D_{3}$ and other parameters. J. Nutr., 103, 586-599.

WILSON A. D., 1963. The effect of diet on the secretion of parotid saliva by sheep. II. Variations in the rate of salivary secretion. Aust. J. agric. Res., 14, 680-689.

YANG M. G., THOMAS J. W., 1965. Absorption and secretion of some organic and inorganic constituents and the distribution of these constituents throughout the alimentary tract of young calves. J. Nutr., 87, 444-458.

YOUNG V. R., LOFGREEN G. P., LUICK J. R., 1966. The effects of phosphorus depletion and of calcium and phosphorus intake, on the endogenous excretion of these elements by sheep. Br. J. Nutr., 20, 795-805.

ZAMYTCHKINA K. S., GRODSENSKY D. E., 1955. The role of radioactive isotopes in investigating the physiology and biochemistry of digestion. Proc. int. Conf. on the Peacef. Uses of Atomic Energy. Geneva, 12, 257-265. 